\title{
Scale and construal: How larger measurement units shrink length estimates and expand mental horizons
}

\author{
Sam J. Maglio • Yaacov Trope
}

Published online: 6 November 2010

(C) Psychonomic Society, Inc. 2010

\begin{abstract}
Scale can vary by requiring a different number of units to measure the same target. But what are the consequences of using fewer, larger units? We draw on past psychophysical research that shows how using fewer units reduces clutter in measurement, translating to shorter length estimates. Additionally, we propose that larger scale is associated with targets further from a person's immediate experience (i.e., psychologically distant) and higher order mental representation. Evidence from Study 1 indicates that framing a target as further away causes it to be estimated as shorter because people use larger units to measure it compared to when the same target is framed as nearby. Two subsequent studies suggest that direct manipulation of larger (versus smaller) measurement scale produces not only shorter length estimates, but also more distal timing judgments (Study 2) and abstract mental representation (Study 3). Implications for scale and level of mental construal are discussed.
\end{abstract}

Keywords Scale $\cdot$ Length estimation · Psychological distance $\cdot$ Construal level theory

Mounting evidence speaks to the fallibility of the perceptual system to provide veridical representations of reality. Instead, undesirable lengths seem shorter (Balcetis \& Dunning, 2007), and people ill-prepared to engage in physical activity overestimate the spaces associated with that activity (Proffitt, Stefanucci, Banton, \& Epstein, 2003). Here, we develop the idea that another factor, scale (operationalized as unit of measurement), may serve as an

S. J. Maglio $(\square) \cdot$ Y. Trope

Department of Psychology, New York University,

6 Washington Place, Room 755B,

New York, NY 10003, USA

e-mail:sam.maglio@nyu.edu additional factor that not only biases length estimation, but also arises from and subsequently impacts the way in which people mentally structure the world at large.

Unit of measurement in length estimation constitutes a specific application of the broader phenomenon of ratio scaling in magnitude estimation (for a review, see Montello, 1991). This is the process by which people use a standard distance (e.g., unit of measurement) to calculate the number of such standards in the magnitude of a test distance (i.e., the target of measurement). Rather than simply an arbitrary parameter, the size of the standard distance has been found in the psychophysical literature to systematically bias length estimates (Baird, 1970; Phipps, 1979; Poulton, 1968). In an investigation into estimated driving distances, Phipps (1979) suggested that larger standard distances (e.g., a standard that is longer than the test distance) serve as cognitive reference points from which people insufficiently adjust in making estimates (see Tversky \& Kahneman, 1974). Consequently, these types of larger standards exaggerate driving distance estimates. In related work, a route-segmentation hypothesis (Allen, 1981; Montello, 1997) predicts that length estimates will be increased when the test distance is partitioned into several separate segments. Thus, using small versus large scale constitutes variation in the standard distance, which segments the test distance into greater or fewer units of measurement, respectively. An important consequence of such numerosity in measurement is the clutter effect, by which the presence of more distinct items increases estimated length (consistent with a route-segmentation hypothesis). For example, Sadalla and Staplin (1980) found that people who crossed several intersections retrospectively estimated the length to be longer than those who had crossed fewer intersections in walking the same absolute length. Similar overestimation of length has been reported for measurement of routes with a 
greater number of major bends (Byrne, 1979) or intervening points (Thorndyke, 1981). Taken together, these findings highlight the relevance of scale in affecting length estimates. However, the precursors to and consequences of using different measurement scales have received far less empirical attention.

The present investigation attempts to fill this gap by drawing upon construal level theory (CLT; Liberman \& Trope, 2008; Trope \& Liberman, 2010), which posits a direct relationship between a person's distance from a target and mental representation of that target. Specifically, as targets become increasingly proximal, people form representations of them based more upon concrete, secondary features (e.g., the color of a car). On the other hand, people represent distal targets in terms of their abstract, primary features (e.g., the function served by a car). Importantly, psychological distancing can occur along different dimensions, including time, geographical space, social distance, and likelihood (Liberman, Trope, \& Stephan, 2007).

Several research findings within the CLT framework are particularly relevant to the present investigation. In one set of studies on unitization, participants were asked to partition a cartoon video into discrete behavioral segments for an event that supposedly took place in a distal (versus proximal) geographical location (Henderson, Fujita, Trope, \& Liberman, 2006). The results indicated that the distal event was segmented into relatively fewer (broader) units. Additionally, the relationship between distance and construal is posited to be bidirectional. Thus, not only do people describe distal events using more abstract language (Fujita, Henderson, Eng, Trope, \& Liberman, 2006), but they also respond to manipulations of high-level construal thinking about abstract elements of an action - by expecting that the action will happen further in the future than when thinking about it concretely (Liberman, Trope, McCrea, \& Sherman, 2007).

Given the evidence relating unitization, psychological distance, and mental construal, we posit a CLT-based explanation for when people will use different measurement units and what patterns of thought will be triggered by differences in scale. First, we predict that distal targets should be subjected to larger scale, accounting for their shorter estimates of length. After testing this prediction of distance influencing choice of unit size, we turn to direct manipulations of measurement unit. Specifically, we test the hypothesis that scale functions as a direct manipulation of level of construal, with large scale prompting more abstract thought. Therefore, relative to smaller scale, large scale should elicit not only shorter length estimates, but also more distal estimates of timing (through the mental association between abstraction and distance) and more high-level categorization of actions (as a direct test of unit size and abstraction).

\section{Study 1}

To begin, we predicted that psychologically distal targets would be measured using larger units than would proximal targets. In turn, the utilization of a larger scale would necessitate fewer units to make a measurement, giving rise to a shorter estimation of length (via differences in the clutter effect). This was tested in Study 1 by allowing participants to create their own unit of measurement for a line meant to represent either a physically proximal or distal path. By measuring the size of unit generated and calculating length estimates for each participant, we expected the relationship between psychological distance and length estimate to be mediated by size of unit created.

\section{Method}

Fifty-five volunteers from the New York University community were approached during lunch in a campus dining center. They were presented with a straight line connecting two points with instructions to imagine that it represented a path they would take to get to a supermarket. As presented, the line measured approximately $160 \mathrm{~mm}$. Psychological distance of the trip was manipulated by providing information about the location of the path. The participants were randomly assigned to believe this trip was to occur in New York (physically proximal; $n=27$ ) or San Francisco (distal; $n=28$ ). They would momentarily estimate the length of this path, but first they had to decide how to perform this task by creating a single unit of measurement with which they would make their estimate. A $140-\mathrm{mm}$ horizontal line was presented on the page, flanked by ' 0 ' on the left and ' 100 ' on the right, and participants were to place a vertical dash on the line to indicate one unit of measurement (with single-unit size starting at the leftmost point on the line and extending to the location of their vertical dash). They then returned to the original line representing the path to the supermarket and measured it by indicating how many of the units they had just created would fit inside it. Finally, participants indicated their age, gender, and familiarity with the metric system to complete the survey.

\section{Results}

As we observed no effects of age, gender, or metric system familiarity in this or any of the studies reported here, these analyses will be omitted. The unit size generated by each participant was measured in millimeters using a ruler, and length estimates were calculated by multiplying unit size by number of units indicated. Participants in the proximal condition generated smaller units of measurement $(M=24.3, \mathrm{SD}=20.9)$ than those 
in the distal condition $(M=39.1, \mathrm{SD}=24.0), t$ $(53)=2.44, p<0.05$. Further, participants in the proximal condition estimated that more of the units they had just created were necessary to measure the length of the line $(M=17.2, \mathrm{SD}=26.0)$ than those in the distal condition $(M=5.2, \mathrm{SD}=3.3), t(53)=2.42$, $p<0.05$. Accordingly, the length estimates we calculated for each participant (unit size multiplied by number of units) were longer for participants in the proximal condition $(M=169, \mathrm{SD}=46)$ than those in the distal condition $(M=140, \mathrm{SD}=38), t(53)=2.47, p<0.05$. To determine whether this difference in length estimation between the distance conditions was due to differential generation of unit sizes, we conducted a mediation analysis using bootstrapping (Preacher \& Hayes, 2004). As predicted, unit size partially mediated the relationship between distance condition and estimated length, $Z=1.94$, $p=0.05$.

\section{Study 2}

The first study established that distal (versus proximal) targets are subjected to larger scale measurements, which in turn reduce length estimates. Here, Study 2 examines the consequences of directly manipulating measurement unit. Past research has shown that targets construed abstractly are mentally represented as being temporally further away (Liberman, Trope, McCrea et al., 2007). If unit of measurement indeed functions as a manipulation of mental construal, the target of measurement should evince an associated distancing from the perceiver. We predict larger scale to yield judgments of not only shorter length, but also timing estimates that are representative of greater psychological (temporal) distance.

\section{Method}

Sixty-nine volunteers in New York City's Bryant Park were presented with a curved line connecting two points and asked to imagine that it represented an upcoming road trip. As presented, the curved line measured approximately $320 \mathrm{~mm}$. The participants were randomly assigned to one of three conditions $(n \mathrm{~s}=23)$, which asked them to estimate the length of the curved line in millimeters, centimeters, or decimeters. The form provided participants with a single-unit sample of their assigned scale (e.g., $1 \mathrm{~cm}$ ) to the side of the curved line. In making their estimates, all participants filled in three blank spaces that were separated by one decimal point; the location of the decimal point varied by condition. For example, the decimal point in the centimeter condition came between the second and third blank spaces: $\mathrm{cm}$. This was done to equate the specificity of estimates made across the conditions.

Next, they assessed when they expected the road trip to happen: a 100-mm horizontal line was presented on the page, flanked by 'very soon' on the left and 'very far from now' on the right. As they were not actually going to embark on a road trip, they were asked to use their general intuition in responding to this question. Participants were to place a vertical dash on the line to make their timing judgment. Finally, participants indicated their age, gender, and familiarity with the metric system to complete the survey.

\section{Results}

The estimates made across conditions were transformed to millimeters prior to analysis. Scale condition significantly influenced estimates of line length, $F(2,66)=11.55$, $p<0.001, \eta^{2}=0.26$. Post hoc analyses (LSD) revealed that estimates made using centimeters $(M=299$, $\mathrm{SD}=93)$ were significantly shorter than those made using millimeters $(M=416, \mathrm{SD}=134)$ and significantly longer than those made using decimeters $(M=203, \mathrm{SD}=66)$, $p s<0.05$. A planned contrast revealed a linear trend of length estimate with finer scale, $F(1,66)=23.02$, $p<0.001$.

Participants' responses to the road trip timing question were measured in millimeters from the leftmost point on the line; as such, larger scores indicate anticipation of the road trip occurring further into the future. Scale condition significantly influenced estimates of trip timing, $F(2$, 66) $=7.79, p=0.001, \eta^{2}=0.19$. Post hoc analyses (LSD) revealed that estimates made using millimeters $(M=22.4, \mathrm{SD}=17)$ led to an expectation that the trip would happen sooner than those made using centimeters $(M=41.6, \mathrm{SD}=24)$ and decimeters $(M=48.7$, $\mathrm{SD}=28), p s<0.01$. A planned contrast revealed a linear trend of trip timing with finer scale, $F(1,66)=14.55$, $p<0.001$. Figure 1 summarizes the results of this study.

\section{Study 3}

The results of Study 2 provided initial evidence for the notion of scale as a construal manipulation, as larger scale led to more distal estimates of road trip timing. However, level of construal can be assessed not only through association to psychological distance (as in Study 2), but also as a general mindset that transfers to new targets independent of the construal manipulation (e.g., Freitas, Gollwitzer, \& Trope, 2004). Thus far, the experimental designs have only considered the consequences of scale for the measured items. With Study 3, we test the carryover 
Fig. 1 Mean length estimates of the curved line and timing estimates of the road trip by scale condition in Study 2. Length estimates are reported in millimeters. Larger timing estimates indicate more anticipated time before taking the road trip. The horizontal dashed line is set at $320 \mathrm{~mm}$, the objectively accurate length of the curved line

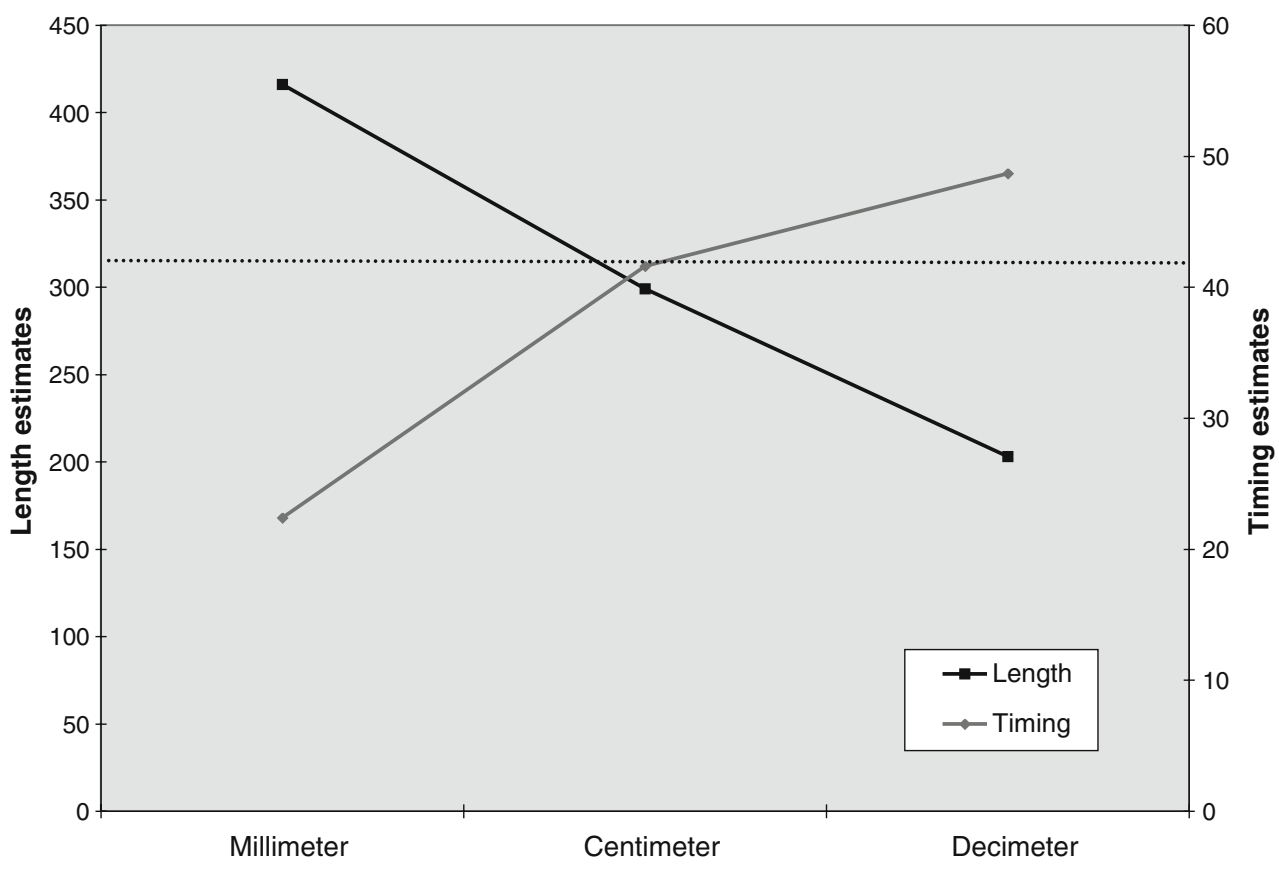

effects of scale to unrelated targets and at the same time operationalize a new methodology to provide a conceptual replication of the earlier studies.

\section{Method}

Fifty-eight New York University students participated in the study in exchange for course credit. They were met by the experimenter and taken to an empty hallway in the psychology department to estimate its length (the actual length was approximately 66 feet). The participants were randomly assigned to one of two conditions $(n \mathbf{s}=29)$, which asked participants to estimate the length of the hallway using either feet or yards. The experimenter presented either a ruler or a yardstick to familiarize participants with their unit of measurement prior to making their length estimation.

After making this estimation, participants were led to a separate room off the hallway to complete a purportedly unrelated packet of questionnaires. These were comprised of a list of 15 actions adapted from the Behavior Identification Form (BIF; Vallacher \& Wegner, 1989), listing specific behaviors and asking participants to choose one of two descriptors to best categorize it: one option pertaining to concrete, specific aspects of the action and the other to abstract, general aspects of it. For example, "making a list" could be described as "writing things down" or "getting organized," respectively. By coding concrete identifications as 0 and abstract identifications as 1 , we were able to calculate an abstract identification index for each participant by summing their scores (which ranged from 0 to 15 , see Fujita, Henderson et al., 2006). Finally, participants indicated their age and gender to complete the study.
Results

The estimates made in yards were transformed to feet prior to analysis. The results indicated that the length estimates made using yards $(M=57, \mathrm{SD}=23)$ were significantly shorter than those made using feet $(M=79, \mathrm{SD}=55), t$ $(56)=2.01, p<0.05$. Further, participants who made their length estimates in yards scored higher on the abstract identification index $(M=8.1, \mathrm{SD}=3.2)$ than those who made their estimates in feet $(M=6.0, \mathrm{SD}=3.1), t$ (56) $=2.59, p<0.05$.

\section{General discussion}

Our three studies suggest that framing a target in terms of a large unit of measurement has implications not only for length estimation, but also for mental representation. In keeping with the tradition of past research, our results indicated that larger unit of measurement produces shorter length estimates (Studies 1-3). Additionally, larger unit of measurement arises as the product of greater spatial distance from a given target (Study 1) and prompts judgments of greater temporal distance (Study 2) and abstract action identification (Study 3).

Taken together, we interpret our results as evidence that scale functions as a manipulation of construal level. Of course, it is not the only means by which to induce construal. Separate investigations have suggested that abstract thought can result from increased psychological distance (described above) as well as positive affect (Gasper \& Clore, 2002) or disfluency (Alter \& 
Oppenheimer, 2008), to sample only two. In an avenue for future work, a mutual association hypothesis would predict that these factors might influence (and be influenced by) scale. Furthermore, our effects offer potential extensions to current research on scale. Furlong and Opfer (2009) report that people are more responsive to financial incentives framed using smaller scale (e.g., 300 cents versus 3 dollars). In their studies, there was only one dimension of value - financial incentive. However, a dichotomy can arise between high and low levels of valuation. The decision to attend a lecture can depend upon both interest in the topic (desirability, a high level feature) and convenience of its timing (feasibility, a low level feature). When these two valuation levels conflict, abstract construal will prompt preference for the option that holds greater high-level value (versus lowlevel value, Liberman \& Trope, 1998). The low-level orientation engendered by using smaller scale may make participants responsive to rewards at this level as opposed to higher order rewards. For instance, describing the length of a cruise as spanning "thousands of miles" rather than "two continents" (small versus large scale, respectively) may prompt concrete representation of it. This could make people more likely to take the cruise only if the ship departs from an easily accessible port (feasibility, a low level feature).

The idea of scale triggering construal provides a potential mechanism for recent empirical findings. Ülkümen, Chakravarti, \& Morwitz (in press) have suggested that coarse- versus fine-grained mental models prompt broader categorization and greater judgment of similarity. Perhaps the coarse-grained models (i.e., large scale) were represented more abstractly, which accounted for the broader categorization and increased similarity (see Liberman, Sagristano, \& Trope, 2002). High-level construal has also been implicated as beneficial for self-control: seeing the situation from a broader context helps to identify the presence of a conflict between long-term goals (e.g., being healthy) and short-term temptations. The result of this process is that the former becomes prioritized, enabling self-control (Fujita, Trope, Liberman, \& Levin-Sagi, 2006). In one study, dieters were first instructed to mark the date on a calendar that either did or did not use a grid to separate the days of the month (narrow or wide frame, respectively). Subsequently, they were offered potato chips, a health-related temptation. The results indicated that those in the wide-frame condition demonstrated greater self-control by eating fewer potato chips than those exposed to the narrow frame (Myrseth \& Fishbach, 2009). The authors report that identification of a self-control conflict accounted for this effect... but what enabled this identification? As the wide-frame condition utilized a larger scale (the salient unit of measurement was months versus days), perhaps this elicited a general pattern of high-level thinking. It could be that this frame of mind promoted the identification of chips as inconsistent with higher order goals, accounting for superior self-control.

In sum, we suggest that the scale applied to a target is far from an incidental factor. Rather, scale gives rise to a specific cognitive orientation - applicable to the original target as well as new targets - toward either the incidental or essential elements of representation. The consequences of this representation bear upon length estimation in addition to psychological distance and level of mental construal. Said differently, large scale leads to big picture thinking.

Acknowledgments We thank Michael Feder, Azeen Popal, and Elle Wang for their support with execution of the studies, and John Franchak and Cris Rabaglia for comments on an earlier version of this manuscript. This research was supported by a NIMH grant no. 5903006A1 to the second author.

\section{References}

Allen, G. (1981). A developmental perspective on the effects of "subdividing" macrospatial experience. Journal of Experimental Psychology: Human Learning and Memory, 7, 120-132.

Alter, A., \& Oppenheimer, D. (2008). Effects of fluency on psychological distance and mental construal (or why New York is a large city, but New York is a civilized jungle). Psychological Science, 19, 161-167.

Baird, J. (1970). Psychophysical analysis of visual space. New York: Pergamon Press.

Balcetis, E., \& Dunning, D. (2007). Cognitive dissonance and the perception of natural environments. Psychological Science, 18, 917-921.

Byrne, R. (1979). Memory for urban geography. The Quarterly Journal of Experimental Psychology, 31, 147-154.

Freitas, A., Gollwitzer, P. M., \& Trope, Y. (2004). The influence of abstract and concrete mindsets on anticipating and guiding others' self-regulatory efforts. Journal of Experimental Social Psychology, 40, 739-752.

Fujita, K., Henderson, M. D., Eng, J., Trope, Y., \& Liberman, N. (2006). Spatial distance and mental construal of social events. Psychological Science, 17, 278-282.

Fujita, K., Trope, Y., Liberman, N., \& Levin-Sagi, M. (2006). Construal levels and self-control. Journal of Personality and Social Psychology, 90, 351-367.

Furlong, E., \& Opfer, J. (2009). Cognitive constraints on how economic rewards affect cooperation. Psychological Science, 20, 11-16.

Gasper, K., \& Clore, G. (2002). Attending to the big picture: Mood and global versus local processing of visual information. Psychological Science, 13, 34-40.

Henderson, M. D., Fujita, K., Trope, Y., \& Liberman, N. (2006). Transcending the "Here": The effect of spatial distance on social judgment. Journal of Personality and Social Psychology, 91, 845-856.

Liberman, N., Sagristano, M., \& Trope, Y. (2002). The effect of temporal distance on level of mental construal. Journal of Experimental Social Psychology, 38, 523-534.

Liberman, N., \& Trope, Y. (1998). The role of feasibility and desirability considerations in near and distant future decisions: 
A test of temporal construal theory. Journal of Personality and Social Psychology, 75, 5-18.

Liberman, N., \& Trope, Y. (2008). The psychology of transcending the here and now. Science, 322, 1201-1205.

Liberman, N., Trope, Y., McCrea, S., \& Sherman, S. (2007). The effect of level of construal on the temporal distance of activity enactment. Journal of Experimental Social Psychology, 43, 143-149.

Liberman, N., Trope, Y., \& Stephan, E. (2007). Psychological distance. In A. W. Kruglanski \& E. T. Higgins (Eds.), Social psychology: Handbook of basic principles (2nd ed., pp. 353381). New York: Guilford Press.

Montello, D. (1991). The measurement of cognitive distance: Methods and construct validity. Journal of Environmental Psychology, 11, 101-122.

Montello, D. (1997). The perception and cognition of environmental distance: Direct sources of information. In S. Hirtle \& A. Frank (Eds.), Spatial information theory: A theoretical basis for gis (pp. 297-311). Berlin: Springer.

Myrseth, K., \& Fishbach, A. (2009). Self-control: A function of knowing when and how to exercise restraint. Current Directions in Psychological Science, 18, 247-252.

Phipps, A. (1979). Scaling problems in the cognition of urban distances. Transactions of the Institute of British Geographers, 4, 94-102.
Poulton, E. (1968). The new psychophysics: Six models for magnitude estimation. Psychological Bulletin, 69, 1-19.

Preacher, K. J., \& Hayes, A. F. (2004). SPSS and SAS procedures for estimating indirect effects in simple mediation models. Behavior Research Methods, Instruments, \& Computers, 36, 717-731.

Proffitt, D., Stefanucci, J., Banton, T., \& Epstein, W. (2003). The role of effort in perceiving distance. Psychological Science, 14, 106112.

Sadalla, E., \& Staplin, L. (1980). The perception of traversed distance: Intersections. Environment and Behavior, 12, 167-182.

Thorndyke, P. (1981). Distance estimation from cognitive maps. Cognitive Psychology, 13, 526-550.

Trope, Y., \& Liberman, N. (2010). Construal-level theory of psychological distance. Psychological Review, 117, 440-463.

Tversky, A., \& Kahneman, D. (1974). Judgment under uncertainty: Heuristics and biases. Science, 185, 1124-1131.

Ülkümen, G., Chakravarti, A., \& Morwitz, V.G. (in press). Categories create mind-sets: The effect of exposure to broad versus narrow categorizations on subsequent, unrelated decisions. Journal of Marketing Research.

Vallacher, R., \& Wegner, D. (1989). Levels of personal agency: Individual variation in action identification. Journal of Personality and Social Psychology, 57, 660-671. 